\title{
Assessing the effects of DER on voltages using a smart meter-driven three-phase LV feeder model
}

DOI:

10.1016/j.epsr.2020.106705

\section{Document Version}

Accepted author manuscript

Link to publication record in Manchester Research Explorer

\section{Citation for published version (APA):}

Wang, Y., Liu, M. Z., \& Ochoa, L. F. (2020). Assessing the effects of DER on voltages using a smart meter-driven three-phase LV feeder model. Electric Power Systems Research, 189, 106705.

https://doi.org/10.1016/j.epsr.2020.106705

\section{Published in:}

Electric Power Systems Research

\section{Citing this paper}

Please note that where the full-text provided on Manchester Research Explorer is the Author Accepted Manuscript or Proof version this may differ from the final Published version. If citing, it is advised that you check and use the publisher's definitive version.

\section{General rights}

Copyright and moral rights for the publications made accessible in the Research Explorer are retained by the authors and/or other copyright owners and it is a condition of accessing publications that users recognise and abide by the legal requirements associated with these rights.

\section{Takedown policy}

If you believe that this document breaches copyright please refer to the University of Manchester's Takedown Procedures [http://man.ac.uk/04Y6Bo] or contact uml.scholarlycommunications@manchester.ac.uk providing relevant details, so we can investigate your claim.

\section{OPEN ACCESS}




\title{
Assessing the Effects of DER on Voltages Using a Smart Meter-Driven Three-Phase LV Feeder Model
}

\author{
Yiqing Wang ${ }^{1}$, Michael Z. Liu ${ }^{1}$, and Luis F. Ochoa ${ }^{1,2}$ \\ ${ }^{1}$ The University of Melbourne, Melbourne, Australia \\ ${ }^{2}$ The University of Manchester, Manchester, United Kingdom
}

\begin{abstract}
The increasing number of residential distributed energy resources (DER) is driving the need for distribution companies to routinely assess potential voltage violations due to excessive power injections. However, this task can be quite challenging in low voltage ( $\mathrm{LV}$ ) feeders due to the limited availability of modelling data. To this end, this work proposes a practical approach to assess the effects of DER on voltages by exploiting historical time-series measurements from smart meters and head of the feeder. First, the impedances of three-phase feeders are determined assuming knowledge of feeder topology and service cable parameters. Then, the resulting smart meter-driven model is used to compute voltages due to potential DER injections. The performance of the proposed methodology is assessed using a realistic Australian three-phase LV feeder with 40 single-phase customers. Results show that, while there is some error in the calculated impedances, customer voltages are sufficiently accurate for DER management purposes.
\end{abstract}

Index Terms-Distributed Energy Resources, DER Impacts, Impedance Estimation, Low Voltage Feeders

\section{INTRODUCTION}

The increasing number of residential distributed energy resources (DER) in low voltage (LV) networks is creating significant operational challenges for distribution companies to manage network issues. For instance, excess generation from PV systems during periods of low demand may cause reverse power flow, and consequently, pushing customer voltages beyond the statutory limits [1]. To ensure customer voltages are maintained within the limits, distribution companies should be able to assess the effects on voltages (and potential violations) due to the intended operation of these DER.

Around the world, more and more LV networks are seeing the deployment of smart meters, measuring active and reactive power as well as voltage magnitudes. However, although the topology of the corresponding feeders might be known, their impedances are, in most cases, not available or accurate. Therefore, three-phase power flow-based calculations to assess the effects of DER are not possible. Consequently, before being able to assess the effects of DER on voltages, it is necessary to first construct an adequate model of the LV feeder, in particular, the impedances (self and mutual).
Although state estimation [2]-[5] is often associated with the calculation of voltages across a given network, its application is to extend observability based on partial measurements and, as such, it requires full knowledge (topology and impedances) of the corresponding circuit. Therefore, this and similar model-based techniques are not applicable when impedances are not known.

Several approaches have been proposed to estimate LV feeder impedances, which depends on availability and type of measurements [6]-[11]. In [6], phasor measurement units (PMUs) are applied at terminal nodes to measure both the phasor and magnitude of bus voltages and currents. However, even though PMUs are common in higher voltage levels (e.g., transmission), their deployment in LV feeders have been limited due to the associated cost [12]. On the other hand, given that there has been significant progress in the deployment of smart meters globally, methods to estimate line impedances from smart meter measurements have also been proposed [7][11]. However, since these works do not consider the mutual coupling between phases as they adopt simple single-phase approaches, and they are not able to cater for the unbalanced nature of real LV feeders.

To this end, this paper proposes a practical approach to assess the effects of DER on voltages using a smart meter-driven three-phase LV feeder model. The impedances of a three-phase $\mathrm{LV}$ feeder are first determined by assuming the knowledge of the feeder topology, customers' phase connections, service cable parameters, and historical measurements from smart meters (single phase) and the head of the feeder (three phase). These assumptions are generally practical: feeder topology and customers' phase connections can be, if not known, estimated using techniques that are showing promising results [13]-[15]; service cable impedances can be known from typical values [8]; and, finally, given that smart meters are becoming common, only one additional device would be required to capture the three-phase data at the head of the LV feeder. Furthermore, given that typical smart meters only capture voltage magnitudes and not the corresponding angles, a linear approximation of the three-phase voltage drop equation is adopted. As the resulting system of equations to solve impedances has more unknowns than equations, a multiple linear regression (MLR) 


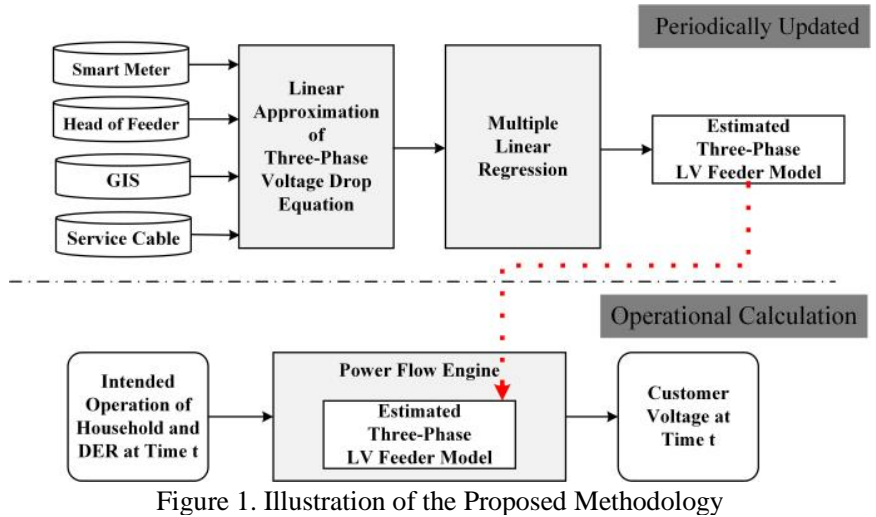

technique [16] is used to exploit historical time-series measurements from smart meters. The estimated impedances are then used to produce a three-phase LV feeder model that, combined with a power flow engine, can be employed for offline or online purposes to assess the effects on customer voltages from any potential DER injection.

The rest of the paper is structured as follows. Section II presents the proposed methodology of assessing the effects of DER on voltages using a smart meter driven three-phase LV feeder model. Section III presents the results of the estimated three-phase LV feeder impedances and customer voltages under different PV penetrations, which is validated on a realistic Australian three-phase LV feeder with 40 single-phase houses. Finally, section IV provides the conclusions of this work.

\section{Methodology}

The proposed methodology includes two parts. The first part aims to estimate three-phase LV feeder impedances, as shown in the upper part of Fig. 1. Four inputs are needed in this process. The first two inputs are the historical measurements from smart meters and the head of the feeder. It includes active power $(P)$ and reactive power $(Q)$ demand as well as voltage magnitude $(V)$. The third input is the topology from the geographic information system (GIS), which includes phase connectivity, lengths and locations of all the elements (e.g., conductors, customers). The last input is the service cable impedances (assumed to be known in the proposed methodology). These four inputs are used to determine the estimated threephase LV feeder model adopting a linear approximation of the three-phase voltage drop equation and the MLR technique. This process is a one-off calculation, which is periodically updated based on the input data. The algorithm corresponding to this process is presented at the end of this section.

Once the LV feeder model is obtained, customer voltages can be assessed at any time (i.e., $t$ ) for any intended household and DER operation (i.e., $\mathrm{P}$ and $\mathrm{Q}$ injections), as shown in the lower part of Fig. 1. The voltages are computed by running a three-phase power flow using the estimated three-phase LV feeder model.

\section{A. Three-Phase Feeder Modelling Considerations}

Fig. 2 illustrates an LV feeder starting from the transformer. It feeds each customer through a three-phase LV feeder (black lines) and a single-phase service cable (red line). The

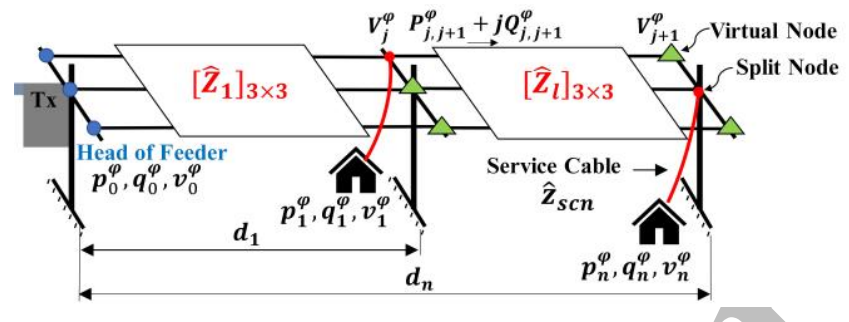

Figure 2. LV Feeder Model

$\mathrm{LV}$ feeder is divided into several segments based on the locations of the split nodes (nodes from which single-phase service cables start). It should be noted that the LV feeder used in this initial work does not consider laterals.

From a modelling perspective, the exact impedance of an LV feeder segment can be expressed in a $4 \times 4$ matrix, which consists of the impedances of the three phases and the neutral. However, to simplify the corresponding model, Kron's reduction [17] is often applied assuming that the neutral wire is connected to ground directly. This results in a $3 \times 3$ impedance matrix, as shown in (1), where $R_{s l}, X_{s l}, R_{m l}$ and $X_{m l}$ represent the self-resistance, self-reactance, mutual resistance, and mutual reactance of the segment $l$. In theory, these four impedance variables are different for each phase. However, to simplify the estimation problem, it is assumed that all three phases share the same impedance variables (i.e., only four impedance variables need to be estimated). Furthermore, given the fact that smart meters only have phase-to-neutral voltages, instead of both phase-to-ground and neutral-to-ground voltages, this reduced model can be adopted.

$$
\left[\hat{Z}_{l}\right]_{3 \times 3}=\left[\begin{array}{ccc}
R_{s l}+j X_{s l} & R_{m l}+j X_{m l} & R_{m l}+j X_{m l} \\
R_{m l}+j X_{m l} & R_{s l}+j X_{s l} & R_{m l}+j X_{m l} \\
R_{m l}+j X_{m l} & R_{m l}+j X_{m l} & R_{s l}+j X_{s l}
\end{array}\right]
$$

Other assumptions need to be made to determine the impedances of all the LV feeder segments following the proposed methodology. This includes:

a) The LV feeder is radial.

b) The feeder topology is known; particularly, phase connections and the lengths of all segments.

c) Single-phase smart meter measurements (active power, $\mathrm{P}$, reactive power, $\mathrm{Q}$, and voltage magnitude, V) from all customers are available.

d) Three-phase measurements $(\mathrm{P}, \mathrm{Q}, \mathrm{V})$ at the head of the feeder are available.

e) Knowledge of the service cable impedances based on typical service cable conductor.

\section{B. Approximation of Three-Phase Voltage Drop Equations}

Equation (2) shows the calculation of the impedance matrix of a given LV feeder segment (in Fig. 3) using Ohm's Law. The voltage drop (or rise) for phase $\varphi$ between $B s_{j}$ and Bus $_{j+1}$ is denoted as $\vec{V}_{j}^{\varphi}-\vec{V}_{j+1}^{\varphi}$. The segment power injection is denoted as $P_{j, j+1}^{\varphi}+j Q_{j, j+1}^{\varphi}$, leaving Bus ${ }_{\mathrm{j}}$ towards Bus $\mathrm{B}_{\mathrm{j}+1}$. 


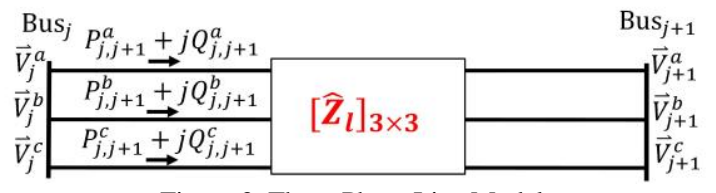

Figure 3. Three-Phase Line Model

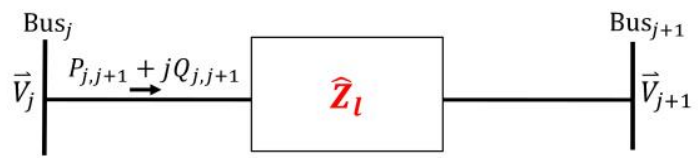

Figure 4. Single-Phase Line Model

$$
\left[\begin{array}{l}
\vec{V}_{j}^{a} \\
\vec{V}_{j}^{b} \\
\vec{V}_{j}^{c}
\end{array}\right]-\left[\begin{array}{l}
\vec{V}_{j+1}^{a} \\
\vec{V}_{j+1}^{b} \\
\vec{V}_{j+1}^{c}
\end{array}\right]=\left[\hat{Z}_{l}\right]_{3 \times 3}\left[\begin{array}{l}
\left(P_{j, j+1}^{a}+j Q_{j, j+1}^{a}\right) / \vec{V}_{j}^{a} \\
\left(P_{j, j+1}^{b}+j Q_{j, j+1}^{b}\right) / \vec{V}_{j}^{b} \\
\left(P_{j, j+1}^{c}+j Q_{j, j+1}^{c}\right) / \vec{V}_{j}^{c}
\end{array}\right]^{*}
$$

However, given that smart meter data is limited to voltage magnitude (i.e., does not include voltage angles), the complex expression in (2) needs to be simplified to an arithmetic expression. To illustrate the proposed simplification process, let us first consider the single-phase line shown in Fig. 4. The complex form of the voltage drop equation for this segment is shown in (3). The current variable $\vec{I}_{j, j+1}$ is also introduced for compactness.

$$
\vec{V}_{j}-\vec{V}_{j+1}=\left(\frac{P_{j, j+1}+j Q_{j, j+1}}{V_{j} \angle \theta_{j}}\right)^{*} \hat{Z}_{l}=\vec{I}_{j, j+1} \hat{Z}_{l}
$$

Assuming a negligible difference in voltage angles between Bus $\mathrm{j}_{\mathrm{j}}$ and Bus $\mathrm{j}_{\mathrm{j}+1}$ (i.e., $\theta_{j} \approx \theta_{j+1}$ ) during normal operating conditions [18], the left-hand side of (3) can be approximated as shown in (4). Subsequently, (5) can be derived from (3) and (4) by separating the real and imaginary parts.

$$
\begin{aligned}
& \vec{V}_{j}-\vec{V}_{j+1}=V_{j} \angle \theta_{j}-V_{j+1} \angle \theta_{j+1} \approx\left(V_{j}-V_{j+1}\right) \angle \theta_{j} \\
& \left\{\begin{array}{l}
\left(V_{j}-V_{j+1}\right) \cos \theta_{j} \approx r_{l} \operatorname{Re}\left\{\vec{I}_{j, j+1}\right\}-x_{l} \operatorname{Im}\left\{\vec{I}_{j, j+1}\right\} \\
\left(V_{j}-V_{j+1}\right) \sin \theta_{j} \approx x_{l} \operatorname{Re}\left\{\vec{I}_{j, j+1}\right\}+r_{l} \operatorname{Im}\left\{\vec{I}_{j, j+1}\right\}
\end{array}\right.
\end{aligned}
$$

For power flow calculation purposes, the reference for the voltage angle is arbitrary. Therefore, for each segment, it is possible to assume $\theta_{j}=0^{\circ}$ for the sending end in (5). Using this assumption, a simplified voltage drop equation containing only voltage magnitudes can be obtained as in (6).

$$
\vec{V}_{j}-\vec{V}_{j+1} \approx V_{j}-V_{j+1} \approx r_{l} \operatorname{Re}\left\{\vec{I}_{l}\right\}-x_{l} \operatorname{Im}\left\{\vec{I}_{l}\right\}
$$

However, while (6) is useful for single-phase calculations, it cannot be directly adopted for three-phase analyses as it does not cater for the coupling between phases. To apply the linear approximation process for the three-phase segment in Fig. 3, let us first take the voltage drop at phase A as an example. Using the same assumption of a negligible voltage angle difference between Bus ${ }_{j}$ and Bus ${ }_{j+1}$, the complex form of the voltage drop equation at phase A according to (2) can be approximated by (7), where $\theta_{j}^{a}, \theta_{j}^{b}$ and $\theta_{j}^{c}$ represent the voltage angles at phases $\mathrm{A}, \mathrm{B}$ and $\mathrm{C}$, respectively.

$$
\begin{aligned}
\left(V_{j}^{a}-V_{j+1}^{a}\right) \angle \theta_{j}^{a} & =\hat{Z}_{s l}\left[\left(P_{j, j+1}^{a}+j Q_{j, j+1}^{a}\right) / V_{j}^{a} \angle \theta_{j}^{a}\right]^{*} \\
& +\hat{Z}_{m l}\left[\left(P_{j, j+1}^{b}+j Q_{j, j+1}^{b}\right) / V_{j}^{b} \angle \theta_{j}^{b}\right]^{*} \\
& +\hat{Z}_{m l}\left[\left(P_{j, j+1}^{c}+j Q_{j, j+1}^{c}\right) / V_{j}^{c} \angle \theta_{j}^{c}\right]^{*}
\end{aligned}
$$

In contrast to the single-phase scenario, information on three voltage angles are needed. As before, a reference angle of $0^{\circ}$ can be adopted for the phase associated with the self-impedance (i.e., phase $\mathrm{A}$ in this case). However, since voltage angles are not measured by the smart meters, it is not possible to deduce the angles of the other two phases (i.e., phases B and $\mathrm{C}$ in this case) purely from smart meter data. To this end, the standard $120^{\circ}$ phase shift for a fully balance system is used instead, i.e., $\theta_{j}^{b}=\theta_{j}^{a}-120^{\circ}$ and $\theta_{j}^{c}=\theta_{j}^{a}+120^{\circ}$. Similarly, for a phase B segment, $\theta_{j}^{b}=0^{\circ}, \theta_{j}^{a}=\theta_{j}^{b}+120^{\circ}$ and $\theta_{j}^{c}=$ $\theta_{j}^{b}-120^{\circ}$, and for a phase $\mathrm{C}$ segment, $\theta_{j}^{c}=0^{\circ}, \theta_{j}^{a}=\theta_{j}^{c}-$ $120^{\circ}$ and $\theta_{j}^{b}=\theta_{j}^{c}+120^{\circ}$.

This approach will introduce some error as real distribution networks are inherently unbalanced. Nonetheless, considering that the segment's mutual impedances are relatively smaller than their self-impedances, the associated error of not knowing the true phase shifts is expected to be negligible.

In summary, for phase $\mathrm{A}$ of a given segment, the final form of the approximated voltage drop equation is shown in (8). The equations for other phases can be derived accordingly and are omitted for compactness.

$$
\begin{aligned}
& \left(V_{j}^{a}-V_{j+1}^{a}\right) \\
& =R_{s l} \operatorname{Re}\left\{\left[\left(P_{j, j+1}^{a}+j Q_{j, j+1}^{a}\right) / V_{j}^{a} \angle 0\right]^{*}\right\} \\
& -X_{s l} \operatorname{Im}\left\{\left[\left(P_{j, j+1}^{a}+j Q_{j, j+1}^{a}\right) / V_{j}^{a} \angle 0\right]^{*}\right\} \\
& +R_{m l} \operatorname{Re}\left\{\left[\left(P_{j, j+1}^{b}+j Q_{j, j+1}^{b}\right) / V_{j}^{b} \angle-120^{\circ}\right]^{*}\right\} \\
& -X_{m l} \operatorname{Im}\left\{\left[\left(P_{j, j+1}^{b}+j Q_{j, j+1}^{b}\right) / V_{j}^{b} \angle-120^{\circ}\right]^{*}\right\} \\
& +R_{m l} \operatorname{Re}\left\{\left[\left(P_{j, j+1}^{c}+j Q_{j, j+1}^{c}\right) / V_{j}^{c} \angle 120^{\circ}\right]^{*}\right\} \\
& -X_{m l} \operatorname{Im}\left\{\left[\left(P_{j, j+1}^{c}+j Q_{j, j+1}^{c}\right) / V_{j}^{c} \angle 120^{\circ}\right]^{*}\right\}
\end{aligned}
$$

Lastly, by following this process for all three phases of each segment, it is possible to obtain a system of three equations (one for each phase) with four unknowns (i.e., $R_{s l}, X_{s l}$, $R_{m l}$ and $X_{m l}$ ). Given the availability of historical time-series measurements from smart meters (i.e., $V_{j}^{\varphi}, V_{j+1}^{\varphi}, P_{j, j+1}^{\varphi}$ and $\left.Q_{j, j+1}^{\varphi}\right)$, the number of equations increases with the number of samples available but the unknowns remain four. This can be exploited by the MLR technique to solve for the unknowns.

\section{Multiple Linear Regression}

The MLR model used in this work is shown in (9), which corresponds to the system of linear equations obtained from the previous sub-section in (8). Following the standard convention of MLR, $\Delta V$ represents the dependent variable, which are the segment voltage drop magnitudes. Furthermore, $\operatorname{Re}\left\{\vec{I}_{s}\right\}$, $\operatorname{Im}\left\{\vec{I}_{s}\right\}, \operatorname{Re}\left\{\vec{I}_{m}\right\}$ and $\operatorname{Im}\left\{\vec{I}_{m}\right\}$ represent the independent variables, which refer to the segment currents. Lastly, indices $\varphi$ and $k$ represent the phase and the time-series sample number, respectively. The large number of samples bring the added benefit of improving overall accuracy. 


$$
\begin{gathered}
\Delta V_{k}^{\varphi}=R_{s l} \operatorname{Re}\left\{\vec{I}_{s}\right\}_{k}^{\varphi}-X_{s l} \operatorname{Im}\left\{\vec{I}_{s}\right\}_{k}^{\varphi}+R_{m l} \operatorname{Re}\left\{\vec{I}_{m}\right\}_{k}^{\varphi} \\
-X_{m l} \operatorname{Im}\left\{\vec{I}_{m}\right\}_{k}^{\varphi}+\epsilon
\end{gathered}
$$

MLR is used to model the relationship between the multiple independent variables and one dependent variable by fitting a linear equation. With these variables as input, MLR results in the best slope coefficients for each independent variable, which are the $R_{s l}, X_{s l}, R_{m l}$ and $X_{m l}$.

\section{Segment Variables}

The methodology requires the voltages and power injections for all phases of each segment. For segments with no customers in one or two phases, the voltages and power injections must be derived. The process is explained below.

Segment Terminal Voltages: Segment terminal voltages can be classified into two different groups: for split nodes (red dots in Fig. 2, which correspond to the start of service cables) and virtual nodes (green triangles in Fig. 2, which are created for the proposed algorithm). Since the service cable impedance is assumed known per assumption e, the split node voltages (denoted as $V_{r}^{\varphi}$ ) can be calculated using the voltage drop equation in (6) and the smart meter data from the corresponding customer. On the other hand, since no customer connections are created from the virtual nodes, the virtual node voltages (denoted as $V_{v}^{\varphi}$ ) are estimated by assuming that the voltage changes linearly with distance between the two adjacent split nodes on either side (i.e., "split node 1" and "split node 2"), as shown in (13). Here, $V_{r 1}^{\varphi}$ and $V_{r 2}^{\varphi}$ represent the voltage at the "split node 1" and the "split node 2 ", and $d_{v}, d_{r 1}$, and $d_{r 2}$ represent the relative distance of the "virtual node", "split node 1" and "split node 2" to the head of the feeder, respectively.

$$
\frac{V_{v}^{\varphi}-V_{r 1}^{\varphi}}{V_{r 2}^{\varphi}-V_{r 1}^{\varphi}}=\frac{d_{v}-d_{r 1}}{d_{r 2}-d_{r 1}} \forall \varphi \in \Phi
$$

Segment Power Injections: The segment power injection at $\mathrm{Bus}_{\mathrm{j}}$ (i.e., $P_{j, j+1}^{\varphi}$ and $Q_{j, j+1}^{\varphi}$ ) is calculated by power balance equations in (14) and (15). Given that the load demand (i.e., $p_{n}^{\varphi}+j q_{n}^{\varphi}$ ) is known, the key is to calculate the segment (i.e., l) active and reactive power losses, denoted as $P_{l}^{l o s s, \varphi}$ and $Q_{l}^{\text {loss, } \varphi}$. The total three-phase LV feeder losses is calculated in (16) and (17). Here, $p_{0}^{\varphi}$ and $q_{0}^{\varphi}$ represent the power at the head of the feeder, and $\sum_{n \in N}\left(p_{n}^{\varphi} / v_{n}^{\varphi}\right)^{2} r_{s c n}$ and $\sum_{n \in N}\left(q_{n}^{\varphi} /\right.$ $\left.v_{n}^{\varphi}\right)^{2} x_{s c n}$ represent the losses of service cables. To determine each segment losses, the total losses are distributed proportionally according to their corresponding voltage drop squared. This assumption will inherently bring error but allows to factor in, to some extent, the corresponding segment losses in the calculation of segment power injections. The voltage drop is denoted as $\Delta V_{l}^{\varphi}$ in (18) and the segment losses are calculated as shown in (19)-(20). The first segment, $l=1$, corresponds to that starting from the head of the feeder.

$$
\begin{gathered}
P_{j-1, j}^{\varphi}-P_{l}^{l o s s, \varphi}=P_{j, j+1}^{\varphi}+p_{n}^{\varphi} \forall j \in J, l \in L, n \in N, \\
\varphi \in \Phi
\end{gathered}
$$

\section{Algorithm: LV Feeder Impedance Estimation}

Input: Smart Meter Measurements $p_{n}^{\varphi}, q_{n}^{\varphi}, v_{n}^{\varphi}, p_{0}^{\varphi}$ $q_{0}^{\varphi}, v_{0}^{\varphi}$

Output: Impedances of all the segments of the feeder: $R_{s l}, X_{s l}, R_{m l}, X_{m l}$

1. Divide the LV feeder into numbers of segments based on the locations of the split nodes.

2. Build three empty three-dimensional matrices with time points, phases and segments for segment terminal voltages $\left(\left[V_{t, \varphi, l}\right]\right)$, segment $P$ injections $\left(\left[P_{t, \varphi, l}\right]\right)$, and segment $\mathbf{Q}$ injections $\left(\left[Q_{t, \varphi, l}\right]\right)$.

3. For each sample $t$ :

Calculate voltages at the split node (start of the service cable) in (6) and put in the $\left[V_{t, \varphi, l}\right]$.

Calculate voltages at "virtual node" in (13) and

End put in $\left[V_{t, \varphi, l}\right]$.

4. For each $t, \varphi, l$ :

Calculate segment $P$ and $Q$ injections in (14)-(20) and complete $\left[P_{t, \varphi, l}\right]$ and $\left[Q_{t, \varphi, l}\right]$.

End

5. For each $t, \varphi, l$ : Input segment terminal voltage magnitude and segment $\mathrm{P}$ and $\mathrm{Q}$ injections to MLR.

End

\section{Run the MLR}

$$
\begin{gathered}
Q_{j-1, j}^{\varphi}-Q_{l}^{\text {loss }, \varphi}=Q_{j, j+1}^{\varphi}+q_{n}^{\varphi} \forall j \in J, l \in L, n \in N, \\
\varphi \in \Phi \\
P_{\text {sum }}^{\text {loss }, \varphi}=p_{0}^{\varphi}-\sum_{n \in N} p_{n}^{\varphi}-\sum_{n \in N}\left(\frac{p_{n}^{\varphi}}{v_{n}^{\varphi}}\right)^{2} r_{\text {scn }} \forall \varphi \in \Phi \\
Q_{\text {sum }}^{\text {loss }, \varphi}=q_{0}^{\varphi}-\sum_{n \in N} q_{n}^{\varphi}-\sum_{n \in N}\left(\frac{q_{n}^{\varphi}}{v_{n}^{\varphi}}\right)^{2} x_{\text {scn }} \forall \varphi \in \Phi \\
\Delta V_{l}^{\varphi}=\left\{\begin{array}{c}
v_{0}^{\varphi}-V_{1}^{\varphi}, l=1 \\
V_{j}^{\varphi}-V_{j+1}^{\varphi}, l \geq 2
\end{array} \forall j \in J, \varphi=\Phi\right. \\
P_{l}^{\text {loss }, \varphi} \approx P_{\text {sum }}^{\text {loss }, \varphi} \times \frac{\left(\Delta V_{l}^{\varphi}\right)^{2}}{\sum_{l \in L}\left(\Delta V_{l}^{\varphi}\right)^{2}} \forall l \in L, \varphi \in \Phi \\
Q_{l}^{\text {loss }, \varphi} \approx Q_{\text {sum }}^{\text {loss } \varphi} \times \frac{\left(\Delta V_{l}^{\varphi}\right)^{2}}{\sum_{l \in L}\left(\Delta V_{l}^{\varphi}\right)^{2}} \forall l \in L, \varphi \in \Phi
\end{gathered}
$$

\section{CASe StUdy}

In this section, the proposed methodology of assessing the effects of DER on voltages using a smart meter driven threephase LV feeder model is tested on a realistic LV feeder from 


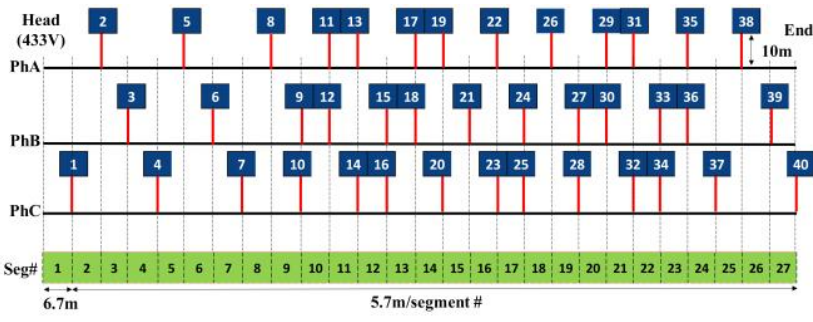

Figure 5. Location and phase connection of the split nodes

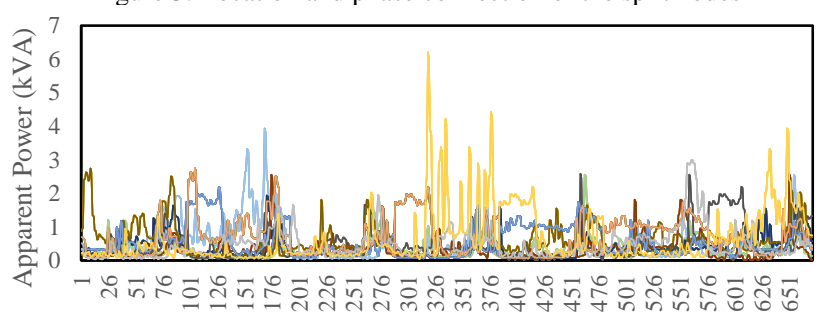

168 Hours (Weekly) - 15 mins resolution

Figure 6. Demand profiles

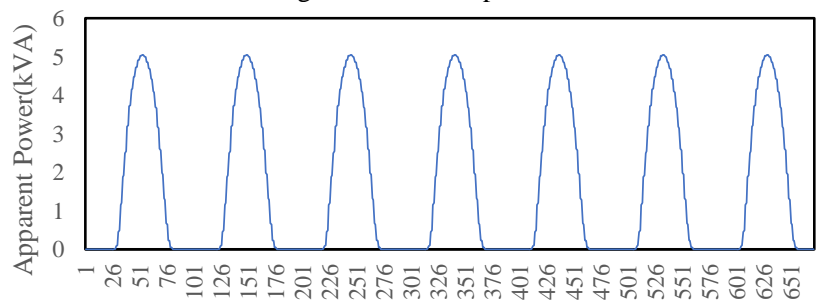

168 Hours (Weekly) - 15 mins resolution

Figure 7. PV generation profile

Victoria, Australia. Firstly, network topology and profiles are presented. Then, the performance of the proposed methodology is presented and discussed.

\section{A. Network Modelling and Profiles}

The studied realistic LV feeder starts from a $22 / 0.433 \mathrm{kV}$ $500 \mathrm{kVA}$ three-phase transformer. The feeder supplies $40 \mathrm{sin}$ gle-phase customers through a total of around $155 \mathrm{~m}$ four-core three-phase 240sq.mm underground cables and a total of $400 \mathrm{~m}$ two-core single-phase 16sq.mm service cables. The split nodes divide the feeder into 27 segments. The relative location and phase connection of the split nodes of single-phase households is shown in Fig. 5. Phases A, B and C have 13, 13 and 14 customers, respectively. Each customer is connected to the feeder with a 10-meter service cable. The length of the first segment (from the transformer to the first split node) is $6.73 \mathrm{~m}$, and the other segments have the same length $(5.7 \mathrm{~m})$. The service cable impedance is $1.15+\mathrm{j} 0.083 \mathrm{~m} \Omega / \mathrm{m}$. For the $\mathrm{LV}$ feeder, the "actual" self and mutual impedances to be estimated are $0.20+j 0.08 \mathrm{~m} \Omega / \mathrm{m}$ and $0.07+j 0.006 . \mathrm{m} \Omega / \mathrm{m}$, respectively.

A pool of 15-min resolution, week-long (i.e., 672 points) anonymized smart meter demand data, as well as, a 15-min resolution, week-long normalized PV generation profile provided by Ausnet Services (a distribution company in Victoria, Australia) is used. The demand profiles for 40 customers are randomly collected from the pool, and a fixed power factor with 0.97 is assumed. Their apparent power is shown in Fig. 6.
Meanwhile, 5.5kWp PV system is considered, and the PV generation is shown in Fig. 7. The distribution system analysis software package OpenDSS [19] (interfaced via Python) is used for three-phase power flow analyses.

\section{B. LV Feeder Impedance Estimation}

Two scenarios are considered to estimate LV feeder models. Scenario 1 estimates the feeder model using 50\% PV penetration, and Scenario 2 estimates the same model using only demand (no PV systems).

For the MLR to calculate the impedance of each segment of the LV feeder, data corresponding to the magnitude of the segment terminal voltages as well as $\mathrm{P}$ and $\mathrm{Q}$ injections from all the three phases during the test period are required. Therefore, for each three-phase segment, 4 parameters per phase are needed each $15 \mathrm{~min}$. This means that the total of $4 \times 3 \times 27 \times$ $672=217,728$ values are sent to the MLR for the whole feeder.

The resulting segment impedances (i.e., $R_{s}, X_{s}, R_{m}$ and $X_{m}$ ) from two estimated LV feeder models are compared with their actual values in Fig. 8 - Fig. 11. Fig. 8 and Fig. 9 illustrate that the self-resistance estimation in Fig. 8 performs better than the self-reactance estimation in Fig.9. Meanwhile, the mutualreactance estimation in Fig. 11 performs better than the mutual-resistance estimation in Fig. 10.

To illustrate the numerical error of the estimated impedances, the two scenarios are compared with their actual values. The minimum, median and maximum absolute percent errors (APEs) are shown in Table I. Scenario 1 results in larger accuracy, compared with that in Scenario 2. In both scenarios, the worst estimated segment is located at the end of the feeder.

Table I Impedance Estimation Error in Two Scenarios

\begin{tabular}{c|c|c}
\hline & Scenario 1 & Scenario 2 \\
\cline { 2 - 3 } & Min/Median/Max APE (\%) & Min/Median/Max APE (\%) \\
\hline $\mathbf{R}_{\mathbf{s}}$ & $0.27 / 0.98 / 91.40$ & $0.39 / 35.41 / 381.77$ \\
\hline $\mathbf{X}_{\mathbf{s}}$ & $0.23 / 7.62 / 938.30$ & $3.79 / 251.14 / 4935.1$ \\
\hline $\mathbf{R}_{\mathbf{m}}$ & $0.19 / 1.09 / 367.58$ & $0.05 / 1.07 / 6397.9$ \\
\hline $\mathbf{X}_{\mathbf{m}}$ & $1.23 / 10.90 / 1184.4$ & $1.05 / 10.80 / 22997$ \\
\hline
\end{tabular}

Overall, Fig. 8-11 show that the line impedances estimated in Scenario 1 are closer to the actual values, compared with the results of Scenario 2. The larger error in Scenario 2 is due to the collinearity between $p_{n}^{\varphi}$ and $q_{n}^{\varphi}$ (i.e., the customer's demand, which are used to compute the inputs to the MLR model) when a fixed power factor is adopted. In general, collinearity is expected to decrease the accuracy of the MLR technique [20]. The level of collinearity is illustrated using (21), where $P_{n, d}$ and $P_{n, g}$ are the customer's demand and PV generation, respectively (the superscripts are dropped for clarity). When there's no PV generation, i.e., $P_{n, g}=0, q_{n}$ is directly correlated with $p_{n}$; consequently, this leads to lower accuracy as seen in Scenario 2. In contrast, when PV generation is high, i.e., $P_{n, g} \gg 0$, the correlation between $p_{n}$ and $q_{n}$ is largely reduced; consequently, this leads to a higher accuracy as seen in 


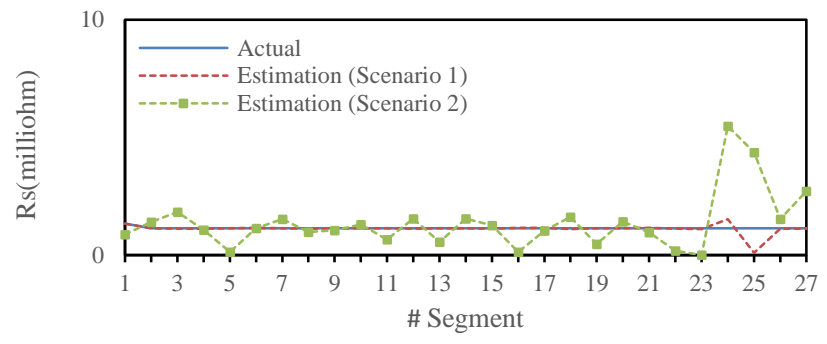

Figure 8. Comparison between the estimation and actual value of the self-re-

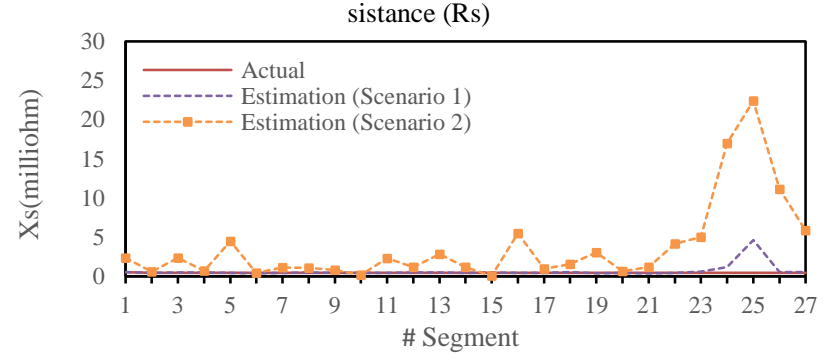

Figure 9. Comparison between the estimation and actual value of the self-reactance $(\mathrm{Xs})$

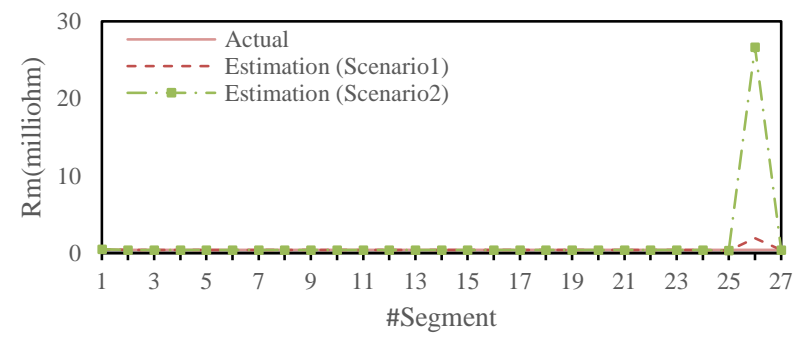

Figure 10. Comparison between the estimation and actual value of the mutual resistance $(\mathrm{Rm})$

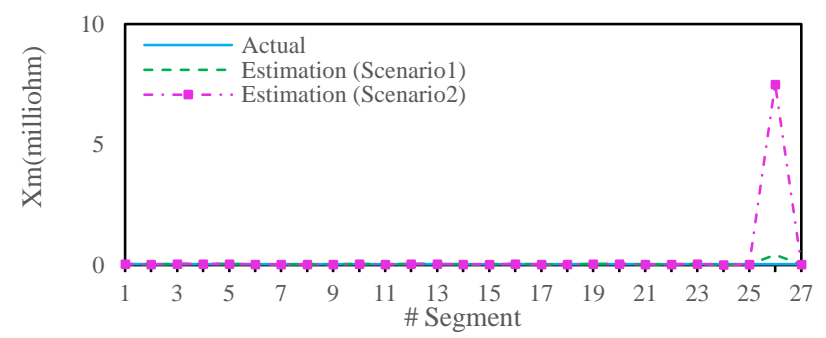

Figure 11. Comparison between the estimation and actual value of the mutual reactance $(\mathrm{Xm})$

Scenario 1 . Therefore, to produce a more accurate LV feeder model in cases where reactive power demand is assumed to be fixed, the historical measurements can be limited to less correlated periods, such as those with higher DER injections. Actual reactive power demand measurements, expected to be largely uncorrelated to active power demand, would also produce more accurate results.

$$
\left\{\begin{array}{c}
p_{n}^{\varphi}=P_{n, d}^{\varphi}-P_{n, g}^{\varphi} \\
q_{n}^{\varphi}=P_{n, d}^{\varphi} \tan \left(\cos ^{-1}(\text { power factor })\right)
\end{array}\right.
$$

\section{Customer Voltage Assessment}

To test the estimated LV feeder models in the calculation of customer voltages, the intended operation of DER is considered to equivalent to $100 \% \mathrm{PV}$ penetration. This corresponds

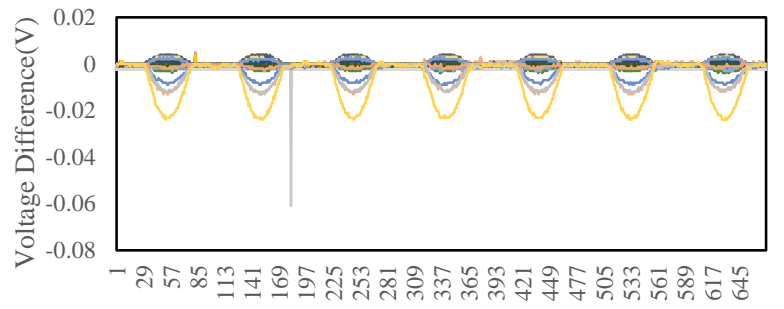

168 Hours (Weekly) - 15 min resolution

Figure 12. Customer Voltage Estimation Mismatch Using the LV Feeder Model Determined in Scenario

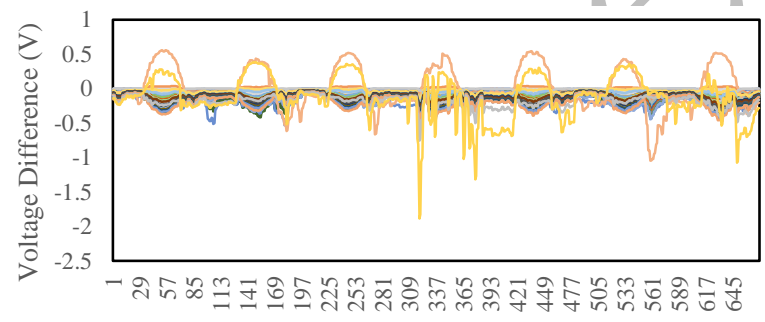

168 Hours (Weekly) - 15 min resolution

Figure 13. Customer Voltage Estimation Mismatch Using the LV Feeder Model Determined in Scenario 2

to significantly different generation conditions/power injections from the scenarios in which the LV feeder models were estimated ( 0 and $50 \% \mathrm{PV}$ penetration). Consequently, voltages are calculated using the three-phase power flow engine OpenDSS, demand/generation from a 100\% PV penetration, and the determined impedances of each scenario when power factor is fixed. Comparisons are carried out on the actual LV feeder model.

The results for the voltage mismatch produced by the LV feeder models using scenarios 1 and 2 are shown in Fig. 12 and Fig. 13, respectively. Positive values correspond to overestimations and negative values to underestimations. In general, both models can accurately estimate customer voltages. The largest mismatch during high PV generation times (largest voltage rise) is around $0.5 \mathrm{~V}$ and corresponds to the model using Scenario 2. However, it is the accuracy of the model using Scenario 1 that stands out. The voltage mismatch is up to 10 times smaller than the mismatch produced by the model using Scenario 2. Therefore, it can be concluded that the LV feeder model estimated using 50\% PV penetration can be used to calculate voltages more accurately.

In addition, in Fig. 12, it can be seen that, during midday, most of the estimated voltages are higher than the actual values (using the actual model), and in a few instances, customer voltages are underestimated. This is due to the self-resistance as it plays a key role in the voltage drop (or rise). The estimated impedances in Scenario 1, shown in Fig. 8 to Fig. 11, are close to or slightly higher than the actual values; particularly before segment 25 . However, the last three segments have lower estimated impedances, which causes, overall, an underestimation of customer voltages during time of high PV generation. The results in Fig. 13 can be explained in a similar fashion. Because the estimated impedances of the last three segments are even higher than in Scenario 1, the calculated voltages tend to have larger overestimations during high PV generation. 


\section{DISCUSSION}

As demonstrated by the results, the accuracy in the calculation of customer voltages depends on the accuracy of the estimated LV feeder impedances. The accuracy of the LV feeder impedances, in turn, depends on the level of correlation among the input variables of the MLR. The more uncorrelated the input variables are, the better the estimated impedances are from the MLR. Therefore, the selection of smart meter data during times of high generation could bring improvements to the impedance estimation part of the proposed methodology.

In addition, from an implementation perspective, distribution companies could speed up and simplify the process of calculating customer voltages by using the equations from the linear approximation of the three-phase voltage drop equation instead of a power flow engine. Furthermore, the algorithm should be extended to cater for laterals (common) as well as the ring operation of $\mathrm{LV}$ feeders (less common).

\section{CONCLUSION}

This paper proposes a practical approach to estimate customer voltages using a smart meter driven three-phase LV feeder model. First, using a linear approximation of the threephase voltage drop equation and the multiple linear regression (MLR) technique, self and mutual impedances of an LV feeder segment are estimated. Then, a three-phase power flow engine and the estimated impedances are used to calculate customer voltages for any demand/generation condition (intended DER operation). The proposed methodology is demonstrated on a realistic Australian three-phase LV feeder with 40 single-phase customers. Two scenarios are used to demonstrate the estimation of the LV feeder model: 0 and $50 \%$ PV penetration. The calculation of customer voltages is demonstrated using those models but with a significantly different generation condition: $100 \%$ PV penetration.

The results show that the $\mathrm{LV}$ feeder model produced using $50 \%$ PV penetration has impedances closer to the actual values. It was found that the accuracy of those impedances is highly dependent on the correlation among the input variables to the MLR; the more uncorrelated the input variables are, in particular active and reactive power demand, the better the estimated impedances. More importantly, the results show that the calculation of voltages using the estimated impedances can be very accurate. In the case study, the largest error was only $0.5 \mathrm{~V}$ during high PV generation times.

In conclusion, the proposed approach can accurately calculate customer voltages for any intended operation of DER in three-phase LV feeders. The use of linear approximations and the MLR makes its implementation more practical than other approaches. Therefore, it has the potential to help distribution companies quantify the effects on customer voltages from different DER operations before they occur, allowing them to take adequate mitigating actions.

\section{APPENDIX}

As demonstrated by the results, the overall accuracy of customer voltages can be considered as high, despite having some errors in the impedances. Nonetheless, it is important to understand how the accuracy of impedance estimation is influenced by the following key assumptions adopted in proposed methodology:

- "Split node" and "Virtual node" voltages in (13);

- Fixed $120^{\circ}$ phase shift; and,

- $\quad$ Line losses in (19)-(20).

To achieve this, the estimated values (based on the proposed methodology) are replaced by their actual values (from the network simulator, i.e., OpenDSS) and impedances are estimated based on Scenario 2 in the following cases:

- $\quad$ Case 1: Actual voltage magnitude;

- Case 2: Actual voltage magnitude and angle; and,

- Case 3: Actual voltage magnitude and angle as well as line losses.

The determined APEs for each case are presented in Table II. Compared to the results in Table I (for Scenario 2), APEs in Table II are significantly smaller. Furthermore, the most significant improvement is from Case 1, i.e., the accuracy of impedance estimation depends more on the correctness of voltage magnitudes than on the accuracy of voltage angles or line losses.

\begin{tabular}{c|c|c|c}
\multicolumn{5}{|c}{ Table II Impedance Estimation Error in Three Cases } \\
\hline & Case 1 & Case 2 & Case 3 \\
\cline { 2 - 4 } & $\begin{array}{c}\text { Min/Median/Max } \\
\text { APE }(\%)\end{array}$ & $\begin{array}{c}\text { Min/Median/Max } \\
\text { APE }(\%)\end{array}$ & $\begin{array}{c}\text { Min/Median/Max } \\
\text { APE }(\%)\end{array}$ \\
\hline $\mathbf{R}_{\mathbf{s}}$ & $0.1 / 1.24 / 47.22$ & $0 / 1.95 / 8.71$ & $0 / 1.5 / 8.26$ \\
\hline $\mathbf{X}_{\mathbf{s}}$ & $0.35 / 13.03 / 480.67$ & $0 / 21.97 / 89.75$ & $0 / 16.68 / 85.30$ \\
\hline $\mathbf{R}_{\mathbf{m}}$ & $0.002 / 0.03 / 13.13$ & $0 / 0.03 / 17.2$ & $0 / 0.03 / 32.43$ \\
\hline $\mathbf{X}_{\mathbf{m}}$ & $0.065 / 0.384 / 35.60$ & $0.0003 / 0.3 / 25.630$ & $0.058 / 0.3 / 51.24$ \\
\hline
\end{tabular}

\section{REFERENCES}

[1] A. T. Procopiou, "Active Management of PV-Rich Low Voltage Networks", Ph.D. dissertation, The University of Manchester, 2017.

[2] H. Yih-Fang, S. Werner, H. Jing, N. Kashyap, and V. Gupta, "State estimation in electric power grids: meeting new challenges presented by the Requirements of the Future Grid," Signal Processing Magazine, IEEE, vol. 29, pp. 33-43, 2012.

[3] A. Mutanen, S. Repo, P. Jarventausta, A. Lof, and D. Della Giustina, "Testing low voltage network state estimation in RTDS environment," in Innovative Smart Grid Technologies Europe (ISGT EUROPE), $20134^{\text {th }}$ IEEE/PES, 2013, pp. 1-5.

[4] A. Abdel-Majeed and M. Braun, "Low voltage system state estimation using smart meters", 2012 47th International Universities Power Engineering Conference (UPEC), $2012 . \quad$ Available: 10.1109/upec.2012.6398598

[5] D. Waeresch, J. Jordan and R. Bischler, "State Estimation in low voltage grids based on smart meter data and photovoltaic-feed-in-forecast", in 23rd International Conference on Electricity Distribution, 2015.

[6] C. M. Roberts, C. M. Shand, K. W. Brady, E. M. Stewart, A. W. McMorran and G. A. Taylor, "Improving distribution network model accuracy using impedance estimation from micro-synchrophasor data," 2016 IEEE Power and Energy Society General Meeting (PESGM), Boston, MA, 2016, pp. 1-5.

[7] S. Iakovlev, R. Evans and I. Mareels, "Low-Voltage Distribution Network Impedances Identification Based on Smart Meter Data", 2018. 
[8] J. Watson, J. Welch and N. Watson, "Use of smart-meter data to determine distribution system topology", The Journal of Engineering, vol. 2016, no. 5, pp. 94-101, 2016, doi: 10.1049/joe.2016.0033.

[9] J. Peppanen, S. Grijalva, M. Reno and R. Broderick, "Distribution system low-voltage circuit topology estimation using smart metering data", 2016, IEEE/PES Transmission and Distribution Conference and Exposition $(T \& D), \quad$ Dallas, TX, 2016, pp. 1-5, doi: 10.1109/TDC.2016.7519985.

[10] S. Park, D. Deka and M. Chcrtkov, "Exact Topology and Parameter Estimation in Distribution Grids with Minimal Observability," in 2018 Power Systems Computation Conference (PSCC), Dublin, Ireland, 2018.

[11] S. Han, D. Kodaira, S. Han, B. Kwon, Y. Hasegawa and H. Aki, "An Automated Impedance Estimation Method in Low-Voltage Distribution Network for Coordinated Voltage Regulation," in IEEE Transactions on Smart Grid, vol. 7, no. 2, pp. 1012-1020, March 2016 , doi: 10.1109/TSG.2015.2489199.

[12] J. De La Ree, V. Centeno, J. S. Thorp, and A. G. Phadke, "Synchronized phasor measurement applications in power systems," IEEE Transactions on Smart Grid, vol. 1, no. 1, pp. 20-27, 2010.

[13] Deka, D., Chertkov, M., Backhaus, S.: 'Topology estimation using graphical models in multi-phase power distribution grids', arXiv preprint arXiv:180306531, 2018.

[14] Y. Liao, Y. Weng, G. Liu, Z. Zhao, C. Tan and R. Rajagopal, "Unbalanced multi-phase distribution grid topology estimation and bus phase identification", in IET Smart Grid, vol. 2, no. 4, pp. 557-570, 2019. doi: 10.1049/iet-stg.2018.0291.

[15] L. Blakely, M. J. Reno, and W. C. Feng, "Spectral Clustering for Customer Phase Identification Using AMI Voltage Timeseries," 2019 IEEE Power Energy Conf. Illinois (PECI), Champaign, IL, USA, 2019, pp. 17, doi: 10.1109/PECI.2019.8698780.

[16] J. Jobson, "Multiple Linear Regression", Springer Texts in Statistics, pp. 219-398, 1991. Available: 10.1007/978-1-4612-0955-3_4

[17] W. Kersting, Distribution system modeling and analysis. Boca Raton, Fla.: Taylor \& Francis, 2007.

[18] E. Lakervi and E. J. Holmes, Electricity Distribution Network Design, 2nd ed. Stevenage, U.K.: Peter Peregrinus, 2003.

[19] R. C. Dugan and T. E. McDermott, "An open source platform for collaborating on smart grid research," 2011 IEEE Power and Energy Society General Meeting, Detroit, MI, USA, 2011, pp. 1-7, doi: 10.1109/PES.2011.6039829.

[20] C. Shalizi, "Lecture 14: Multiple Linear Regression", Stat.cmu.edu, 2015. [Online]. Available: https://www.stat.cmu.edu/ cshalizi/mreg/15/lectures/14/lecture-14.pdf.

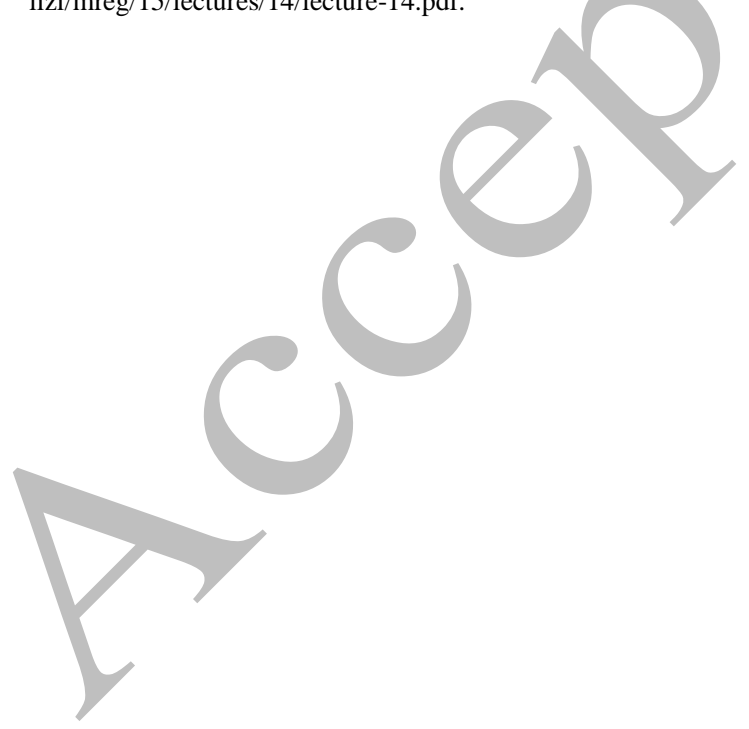

